higher DAS-28/SDAI/CDAI/RAPID-3, lower rate of ACR/EULAR Boolean criteria remission, use of higher dose of glucocorticoid, and higher rate of bone erosion not only on foot but also hand X-rays. Among those patients, 174 patients $(8.5 \%)$ were in DAS 28 clinical remission. Twenty-one of 174 patients (12.1\%) had foot arthritis, who showed higher swollen and tender joint count, RAPID-3 score, and patients' global assessment but not physicians' global assessment than those without foot arthritis. Among patients with foot arthritis, rate of complete remission was the highest in patients with CDAI $(66.7 \%)$.

Conclusions: In patients with rheumatoid arthritis, foot and/or ankle arthritis is associated with high disease activity, not achieving complete remission despite of various clinical remission criteria and discordance between patients' and physicians' global assessment.

Disclosure of Interest: None declared

DOI: 10.1136/annrheumdis-2018-eular.4525

\section{AB0310 USING TREAT-TO-TARGET STRATEGY BY DETERMINING PHYSICAL DISABILITY AND GLUCOCORTICOID REDUCTION STRONGLY INFLUENCE FUNCTIONAL REMISSION IN RHEUMATOID ARTHRITIS}

T. Hagiwara, K. Kamada, N. Namura. Rheumatology, Takarazuka City Hospital, Takarazuka, Japan

Background: The initial target in the treatment of rheumatoid arthritis $(R A)$ is to achieve clinical remission (CR) through Boolean definition and/or index-based criteria and sustain $\mathrm{CR}$, and the final target is to maximise long-term health-related quality of life (HRQoL) through arthritis control, joint damage prevention, function normalisation and social participation. ${ }^{1}$

Objectives: We aimed to determine the factors that inhibit the achievement of functional remission $(\mathrm{FcR})$ in terms of HRQoL.

Methods: A total of 227 patients with RA who had underwent first treatment between October 2014 and December 2017 and had not changed/added another disease-modifying anti-rheumatic drugs (DMARDs) for 12 weeks before the observation day were examined. We used daily-life function and social activity participation to evaluate HRQoL. We adopted the Health Assessment Questionnaire Disability Index (HAQDI) as daily-life functional assessment and EuroQoL 5 dimensions -5 levels (EQ5D) for health status with considerable potential assessment. FcR was defined in this study as HAQDI $\leq 0.5$ and EQ5D $\geq 0.867$ " 0.867 is the lowest QoL score when only one category permits $\leq 2$ of the 5 levels, but others need 1 in all five categories". We investigated their age at RA onset, sex, Steinbrocker stage and functional class, HAQDI, disease activity level, rheumatoid factor, anti-cyclic citrullinated peptide antibody at the first consultation, age, disease activity level, HAQDI, EQ5D, and status of methotrexate (MTX), glucocorticoids (GCs) and biologic/target synthetic DMARD (b/ts-DMARD) use at the last observational day. First, the assumed remissions were analysed using the $\mathrm{FcR}$ as a purpose variable for these factors. Subsequently, the odds ratio and $95 \%$ confidence interval $(95 \% \mathrm{Cl})$ were examined using multiple logistic regression analysis for the statistically significantly different factors and risk factors.

Results: The CR achievement rate at the last observational day by Boolean definition and Simple Disease Activity Index were $40.5 \%$ and $50.2 \%$, respectively. The achievement ratio of $\mathrm{HAQ}-\mathrm{DI} \leq 0.5$, EQ5D $>0.867$ and $\mathrm{FcR}$ was $73.1 \%$, $48.5 \%$, and $46.7 \%$, respectively. The differences in disease duration, stage, class and HAQDI at the time of the first interview; state of MTX, GCs and b/ts-DMARD use; and age at the last observational day for the achievement of FcR were statistically significant. The odds ratios, as determined by multiple logistic regression analysis of the above-mentioned results, were $1.034(95 \% \mathrm{Cl} 0.982-1.088$, $\mathrm{p}=0.202$ ) for disease duration (per 1 year), $1.576(95 \% \mathrm{Cl} 1.257-1.977, \mathrm{p}<0.001)$ for HAQDI at first interview (per 0.5), $0.615(95 \% \mathrm{Cl} 0.277-1.365, \mathrm{p}=0.232$ ) and $4.943(95 \% \mathrm{Cl} 1.683-14.524, \mathrm{p}<0.01)$ for GCs state (non-use vs temporal use and non-use vs continuous use, respectively), and 1.164 (95\% Cl 1.037-1.307, $\mathrm{p}<0.05)$ for age at last observational day.

Conclusions: HRQoL is important as they will influence future treatment strategy. Our results indicated the importance of functional assessment at first interview and demonstrated how to use GCs for the treatment of RA. Ageing always contributes to patients' frailty, and it is unavoidable it. To achieve FcR, functional assessment should be performed during the first interview and short-term use of $\mathrm{GC}$ is useful for prompt functional recovery, in consideration of ageing.

REFERENCE:

[1] de Wit MP, et al. ARD 2011;70(6):891-895. doi:10.1136/ard.2010.14662

Disclosure of Interest: None declared

DOI: 10.1136/annrheumdis-2018-eular.2810

\section{$\mathrm{AB} 0311$ \\ DETERMINANTS OF NON-NOCICEPTIVE PAIN IN RHEUMATOID ARTHRITIS}

T.M. Rocha, S. Pimenta, M. Bernardes, A. Bernardo, M. Barbosa, R. Lucas, L. Costa. Rheumatology, Centro Hospitalar de São João, Porto, Portugal

Background: A neuropathic component (NP) of Rheumatoid Arthritis (RA) pain was described in nearly a third of the patients. Radiographic damage is a reflection of cumulative disease activity and other pathophysiological processes. Some clinical predictors of RA NP were recently identified by our group, but association and adjustment for radiographic damage were not studied.

Objectives: To estimate the clinical predictors of NP in RA patients adjusting for their radiographic damage.

Methods: Cross-sectional study was performed with RA patients followed at our Rheumatology department. Patients with diagnosed neuropathy or non-RA risk factors for NP were excluded. Selected patients were evaluated in a medical visit. Demographic and clinical data were collected and two questionnaires were applied to assess NP: the Leeds Assessment of Neuropathic Symptoms (LANSS) and the painDETECT (PDQ). Wrists, hands and feet radiographic studies from the previous 12 months were classified according to the modified van der Heijde Sharp's method by one trained reader, blinded for patient clinical variables and treatment allocation. Univariate and multivariate logistic regression were performed adjusting for global radiographic score (GS). Significance level was set as $<0.05$.

Results: Ninety one RA patients were included. Seventy (77\%) were women, with a mean (SD) age of 55.6 (10.8) years and median disease duration of 12 years, ${ }^{2-41} 84 \%$ patients were seropositive for Rheumatoid Factor and/or ACPA $85(93 \%)$ were treated with DMARDs and $41 \%$ with a biological DMARD (bDMARDs). The mean (SD) DAS28 4V CRP was 3.15 (0.77). The median join erosion score was 28 (range: $3-143$ ) and the median joint space narrowing (JN) was 46 (range: $10-133$ ). Forty-two (46\%) patients had LANSS NP ( $\geq 12)$ and $29 \%$ had a possible/likely NP in the PDQ $(>12)$. JN was a significant negative predicto of LANSS NP (OR: 0.98, $\mathrm{p}=0.02$ ). After adjusting for $\mathrm{GS}$, gender was not associated with NP. Pain VAS, patient global activity and the tender joint count were positive predictors of NP by both tests. Swollen joint count, ESR or CRP levels were not significantly associated with NP. DAS28 CRP was a significant positive predictor of NP by both tests (OR 1.89 for LANSS and OR: 2.06 for PDQ, $p<0.05$ ); as well as the HAQ score (OR: 2.68 and OR: 4.85, respectively, $p<0.05$ ). Positivity for ACPA was a negative predictor of LANSS NP (OR: 0.31, $p=0.048$ ), as previously described. Current methotrexate treatment had lower odds of LANSS NP (OR: $0.35, p=0.04$ ) but did not remained significant after adjustment for DAS28 CRP. Previous/current Hydroxychloroquine (HCQ) treatment was once more a negative predictor for PDQ NP (OR: $0.11, p=0.04$ ) and remained significant after adjustment for DAS28 CRP. Previous/current leflunomide (LFN) was newly a positive predictor of NP in both tests (OR: 3.41 for LANSS and OR: 2.95 for PDQ, $\mathrm{p}<0.05$ ), persisting after disease activity adjustment for LANSS NP. No other associations were found.

Conclusions: Consistently with our previous data, this study supports an association between NP and disease activity/functional scores but not with objective inflammatory measures. Possible increased risk of NP in LFN treated patients was newly pointed and protective role of ACPA positivity and HCQ was reinforced.

\section{REFERENCES}

[1] Christensen AW, et al. Scand J Rheumatol 2016;1-9.

[2] Martins-Rocha T, et al. Ann Rheum Dis, 2017;76(suppl 2):1177.

Disclosure of Interest: T. Rocha Grant/research support from: Portuguese Society of Rheumatology/Alfa Wassermann on May 2015, S. Pimenta: None declared, M. Bernardes: None declared, A. Bernardo: None declared, M. Barbosa: None declared, R. Lucas: None declared, L. Costa: None declared DOI: 10.1136/annrheumdis-2018-eular.5769

\section{AB0312 INFLAMMATORY ACTIVITY APPEARS WELL CONTROLLED IN MOST PATIENTS WITH RHEUMATOID ARTHRITIS (RA) IN CONTEMPORARY RHEUMATOLOGY CARE, BUT JOINT DAMAGE AND DISTRESS REMAIN AS PROBLEMS OF GREATER MAGNITUDE THAN INFLAMMATION}

T. Pincus, I. Castrejon, J.A. Block. Rheumatology, Rush University Medical Center, Chicago, USA

Background: Rheumatologists traditionally use quantitative measures such as swollen and tender joint counts and laboratory tests to assess inflammatory activity. However, structural damage to joints, as well patient distress seen as fibromyalgia, depression, etc., may be important clinical problems for many RA patients, but are described narratively in the medical record rather than estimated 